\title{
Evaluation of anti-tubercular activity of nicotinic and isoniazid analogues
}

\author{
Maria C. S. Lourenço, ${ }^{a}$ Marcus V. N. de Souza, ${ }^{b^{*}}$ Alessandra C. Pinheiro, \\ Marcelle de L. Ferreira, ${ }^{b}$ Raoni S. B. Gonçalves, ${ }^{\text {b }}$ Thais Cristina M. Nogueira, \\ and Mônica A. Peralta ${ }^{b}$ \\ ${ }^{a} I P E C-I n s t i t u t o$ de Pesquisas Clínicas Evandro Chagas, Departamento de Bacteriologia \\ Av. Brasil, 4365 -Manguinhos, Rio de Janeiro - RJ, Brazil \\ ${ }^{b}$ FioCruz-Fundação Oswaldo Cruz, Instituto de Tecnologia em Fármacos-Far-Manguinhos \\ Rua Sizenando Nabuco, 100, Manguinhos, 21041-250 - Rio de Janeiro-RJ \\ E-mail: marcos souza@far.fiocruz.br
}

\begin{abstract}
Several nicotinic and isoniazid derivatives, most of them containing nitro groups were synthesized by our group, and evaluated for their in vitro antibacterial activity against Mycobacterium tuberculosis $\mathrm{H}_{37} \mathrm{Rv}$ using the Alamar Blue susceptibility test and the activity expressed as the minimum inhibitory concentration (MIC) in $\mathrm{mg} / \mathrm{mL}$. The compound 11d exhibited the best result $(1.2 \mu \mathrm{g} / \mathrm{mL})$ when compared with first line drugs such as isoniazid (INH) and rifampicin (RIP). Therefore this class of compounds could be a good starting point to develop new lead compounds in the treatment of multi-drug resistant tuberculosis.
\end{abstract}

Keywords: Tuberculosis, isoniazid, nicotinic acid, analogs

\section{Introduction}

The pyridine nucleus is an important heteroaromatic class of compounds with a wide range of activities and it is present in many products such as drugs, vitamins, food, flavorings, plants, dyes, rubber products, adhesives, insecticides and herbicides ${ }^{1}$. In this context, nicotinic acid (pyridine-3-carboxylic acid), also known as niacin and vitamin B3, is found in various plants and animals and has vital roles in such biological processes as production of energy, signal transduction, regulation of gene expression and synthesis of fatty acids, cholesterol and steroids $^{2,3}$. Nicotinic acid derivatives and its isomers have also been investigated as an agent for the prevention or delay of the onset of type 1 diabetes mellitus ${ }^{4}$. They also have anti-bacterial, anti-oxidant, anti-inflammatory and anti-carcinogenic activities, and have putative activity against osteoarthritis and granuloma annulare ${ }^{3}$. For example, it can be mentioned the importance 
of anti-tuberculosis first-line drug isoniazid ${ }^{5}$, which is an analogue of isonicotinic acid, an isomer of nicotinic acid. Nicotinic acid derivatives are also an important start material for the preparation of other biological activity compounds. Considering that, 2-chloro-3pyridinecarbonyl chloride is a useful intermediate for preparation of Nevirapine (Viramune, 1), a valuable anti-AIDS drug ${ }^{6}$. Due to the importance of nicotinic acid derivatives and its isomers, the aim of this article is to evaluate the in vitro antibacterial activity against Mycobacterium tuberculosis of several compounds of this class synthesized by our group.

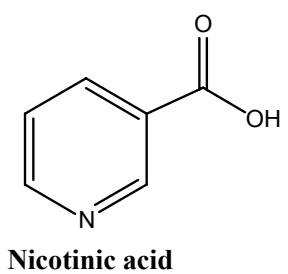

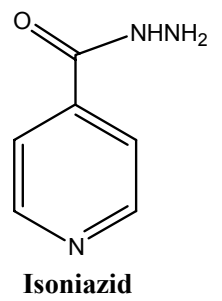

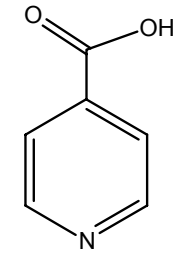

Isonicotinic acid

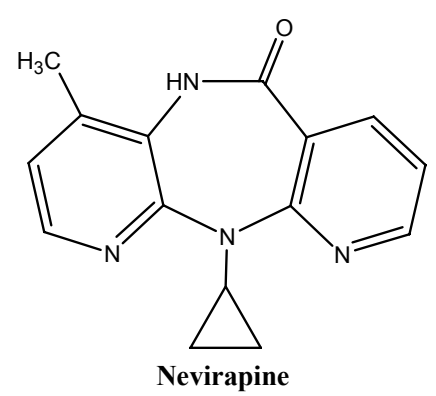

(Viramune)

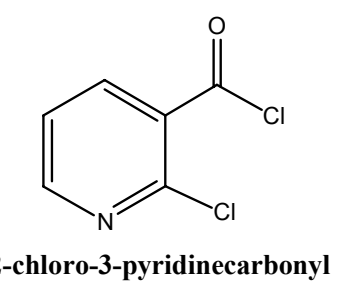

chloride

\section{Figure 1}

\section{Results and Discussion}

\section{Nitro nicotinic derivatives}

The synthesis of nitro compounds described in the schemes (1-4) was based on the reaction between 2-chloro-3-pyridinecarbonylchloride and nitro anilines or nitro phenylhydrazines, leading to the compounds $\mathbf{2 a - c},{ }^{7} \mathbf{4 a - g},{ }^{8} \mathbf{6 a - c},{ }^{9} \mathbf{8 a - d},{ }^{10}$ and $\mathbf{9 a - d ^ { 1 0 }}$ or the coupling of nitro acid chlorides or aldehydes with isoniazid, leading to the products 11a-d ${ }^{11}$. Nowadays, important attention has been payed for nitro compounds as anti-TB, especially due to the discovery of the compound nitroimidazole PA-824 (Figure 2) ${ }^{5}$, which has shown important TB activity against MDR-TB strains, promising activity in latent state TB and potent sterilization effects compared with those of rifampicin and isoniazid. Another advantage of PA-824 is that no significant inhibition of cytochrome P450 isozymes has been observed suggesting that it could be used with HIV medications, being useful in the TB/HIV treatment. Due to its promising activity against TB, the nitroimidazole PA-824 is under clinical phase studies being developed by TB Alliance. 


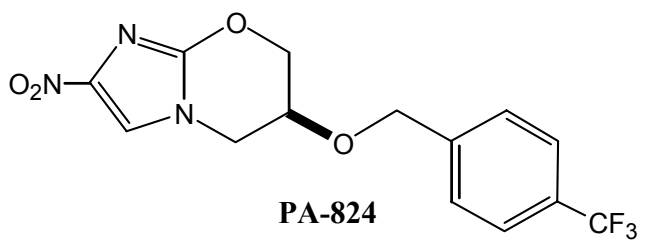

Chiron Corporation, TB Alliance

\section{Figure 2}

\section{1. $N$-Nitro-2-chloronicotinamides}

The $N$-nitro-2-chloronicotinamides, as well others $N$-aryl-2-chloronicotinamides (Schemes 1 and 2) were synthesized and evaluated for their in vitro antibacterial activity against Mycobacterium tuberculosis $\mathrm{H}_{37} \mathrm{Rv}$ using the Alamar Blue susceptibility test and the activity expressed as the minimum inhibitory concentration (MIC) in $\mathrm{mg} / \mathrm{mL}$. However, none of the compounds above mentioned were active. The compounds $\mathbf{2 a - c}$ and $\mathbf{4 a - g}$ were identified by spectral data, which are presented in the Tables 1-3.

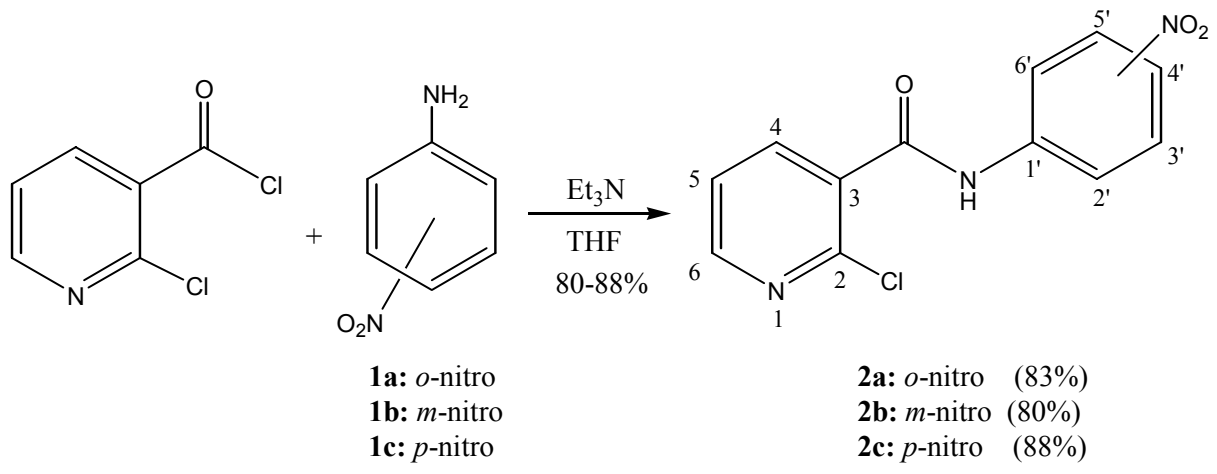

\section{Scheme 1}<smiles>O=C(Cl)c1cccnc1Cl</smiles>

2-chloro-3pyridinecarbonyl chloride<smiles>[R]c1ccc(N)cc1</smiles>

3a: $\mathrm{R}=\mathrm{H}$

3c: $\mathrm{R}=\mathrm{Br}$

3e: $\mathrm{R}=\mathrm{Cl}$ 3g: $\mathrm{R}=\mathrm{CN}$

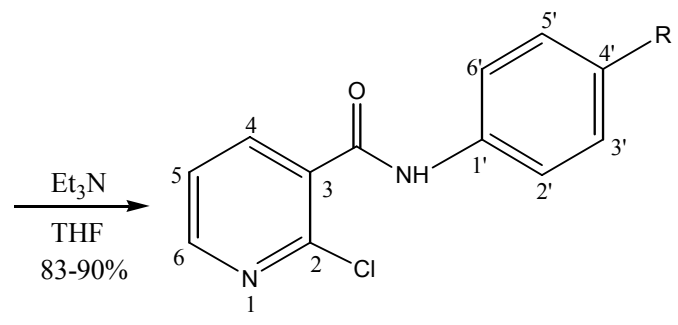

3b: $\mathrm{R}=\mathrm{OMe}$ 3d: $\mathrm{R}=\mathrm{I}$ 3f: $R=F$

4a: $\mathrm{R}=\mathrm{H} \quad(90 \%)$ 4c: $\mathrm{R}=\mathrm{Br} \quad(88 \%)$ 4e: $\mathrm{R}=\mathrm{Cl} \quad(85 \%)$ 4g: $\mathrm{R}=\mathrm{CN} \quad(83 \%)$ 4b: $\mathrm{R}=\mathrm{OMe}(85 \%)$

4d: $\mathrm{R}=\mathrm{I} \quad(84 \%)$

4f: $\mathrm{R}=\mathrm{F} \quad(88 \%)$

Scheme 2 
Table 1. Data of compounds 2a-c and 4a-g

\begin{tabular}{ccccccc}
\hline \multirow{2}{*}{ Products } & \multirow{2}{*}{ Formula } & \multirow{2}{*}[\mathrm{M}]{$^{+}$} & \multirow{2}{*}{$\mathrm{PF}\left({ }^{\circ} \mathrm{C}\right)$} & \multirow{2}{*}{ Yield (\%) } & \multicolumn{2}{c}{$\mathrm{IR}\left(\mathrm{cm}^{-1}\right)$} \\
\hline $\mathbf{2 a}$ & $\mathrm{C}_{12} \mathrm{H}_{8} \mathrm{ClN}_{3} \mathrm{O}_{3}$ & 277 & $157-159$ & 83 & 1687 & 3327 \\
$\mathbf{2 b}$ & $\mathrm{C}_{12} \mathrm{H}_{8} \mathrm{ClN}_{3} \mathrm{O}_{3}$ & 277 & $155-158$ & 80 & 1672 & 3358 \\
$\mathbf{2 c}$ & $\mathrm{C}_{12} \mathrm{H}_{8} \mathrm{ClN}_{3} \mathrm{O}_{3}$ & 277 & $206-208$ & 88 & 1649 & 3266 \\
$\mathbf{4 a}$ & $\mathrm{C}_{12} \mathrm{H}_{9} \mathrm{ClN}_{2} \mathrm{O}$ & 232 & $122-124$ & 90 & 1662 & 3250 \\
$\mathbf{4 b}$ & $\mathrm{C}_{13} \mathrm{H}_{11} \mathrm{ClN}_{2} \mathrm{O}_{2}$ & 262 & $110-111$ & 85 & 1655 & 3235 \\
$\mathbf{4 c}$ & $\mathrm{C}_{12} \mathrm{H}_{8} \mathrm{BrClN}_{2} \mathrm{O}$ & 312 & $126-127$ & 88 & 1649 & 3266 \\
$\mathbf{4 d}$ & $\mathrm{C}_{12} \mathrm{H}_{8} \mathrm{ClIN}_{2} \mathrm{O}$ & 358 & $175-177$ & 84 & 1645 & 3221 \\
$\mathbf{4 e}$ & $\mathrm{C}_{12} \mathrm{H}_{8} \mathrm{Cl}_{2} \mathrm{~N}_{2} \mathrm{O}$ & 267 & $148-149$ & 85 & 1658 & 3280 \\
$\mathbf{4 f}$ & $\mathrm{C}_{12} \mathrm{H}_{8} \mathrm{ClFN}_{2} \mathrm{O}$ & 250 & $93-94$ & 88 & 1661 & 3135 \\
$\mathbf{4 g}$ & $\mathrm{C}_{13} \mathrm{H}_{8} \mathrm{ClN}_{3} \mathrm{O}$ & 257 & $172-173$ & 83 & 1688 & 3261 \\
\hline
\end{tabular}

Table 2. ${ }^{1} \mathrm{H}-\mathrm{NMR}$ chemical shifts of compounds $\mathbf{2 a - c}$ and $\mathbf{4 a - g}$

\begin{tabular}{cccccccc}
\hline & \multicolumn{7}{c}{$\delta{ }^{1} \mathrm{H}(\mathrm{ppm})$} \\
\hline Products & $\mathrm{NH}$ & $\mathrm{H}_{4}$ & $\mathrm{H}_{5}$ & $\mathrm{H}_{6}$ & $\mathrm{H}_{2^{\prime},} 6^{\prime}$ & $\mathrm{H}_{3^{\prime}, 5^{\prime}}$ & $\mathrm{H}_{4^{\prime}}$ \\
$\mathbf{2 a}$ & 11.06 & 7.67 & 7.62 & 8.58 & $--; 8.02$ & $8.06,7.49$ & 7.78 \\
$\mathbf{2 b}$ & 11.15 & 8.02 & 7.61 & 8.58 & $8.74,8.00$ & $--; 7.69$ & 8.15 \\
$\mathbf{2 c}$ & 11.27 & 8.17 & 7.62 & 8.59 & 7.97 & 8.30 & -- \\
$\mathbf{4 a}$ & 10.63 & 8.08 & 7.56 & 8.54 & 7.71 & 7.37 & 7.14 \\
$\mathbf{4 b}$ & 10.50 & 8.06 & 7.55 & 8.53 & 7.63 & 6.95 & -- \\
$\mathbf{4 c}$ & 10.79 & 8.10 & 7.59 & 8.55 & 7.57 & 7.70 & -- \\
$\mathbf{4 d}$ & 10.75 & 8.10 & 7.56 & 8.55 & 7.55 & 7.72 & -- \\
$\mathbf{4 e}$ & 10.79 & 8.11 & 7.58 & 8.55 & 7.44 & 7.75 & -- \\
$\mathbf{4 f}$ & 10.71 & 8.10 & 7.58 & 8.55 & 7.22 & 7.74 & -- \\
$\mathbf{4 g}$ & 11.16 & 8.15 & 7.60 & 8.57 & 7.86 & 7.89 & -- \\
\hline
\end{tabular}


Table 3. ${ }^{13} \mathrm{C}$-NMR chemical shifts of compounds $2 \mathbf{a}-\mathbf{c}$ and $\mathbf{4 a - g}$

\begin{tabular}{ccccccccccc}
\hline & \multicolumn{10}{c}{$\delta(\mathrm{ppm})$} \\
\hline Products & $\mathrm{C}_{2}$ & $\mathrm{C}_{3}$ & $\mathrm{C}_{4}$ & $\mathrm{C}_{5}$ & $\mathrm{C}_{6}$ & $\mathrm{C}=\mathrm{O}$ & $\mathrm{C}_{1^{\prime}}$ & $\mathrm{C}_{2^{\prime}, 6^{\prime}}$ & $\mathrm{C}_{3^{\prime}, 5^{\prime}}$ & $\mathrm{C}_{4^{\prime}}$ \\
\hline $\mathbf{2 a}$ & 146.5 & 131.8 & 138.3 & 123.1 & 151.0 & 163.6 & 133.9 & $130-126.3$ & $126-124.9$ & 143.2 \\
$\mathbf{2 b}$ & 146.3 & 132.4 & 138.2 & 123.1 & 150.8 & 164.0 & 139.6 & $119-113.6$ & $131-125.5$ & 147.9 \\
$\mathbf{2 c}$ & 146.3 & 132.4 & 138.3 & 123.2 & 150.9 & 164.2 & 142.8 & 119.4 & 125.0 & 144.6 \\
$\mathbf{4 a}$ & 146.4 & 133.1 & 138.1 & 123.1 & 150.4 & 163.4 & 138.1 & 119.5 & 128.8 & 124.0 \\
$\mathbf{4 b}$ & 146.5 & 131.8 & 138.1 & 123.1 & 150.3 & 163.1 & 133.3 & 114.0 & 121.1 & 155.8 \\
$\mathbf{4 c}$ & 146.4 & 132.9 & 138.2 & 123.1 & 150.6 & 163.6 & 138.0 & 121.5 & 131.7 & 115.8 \\
$\mathbf{4 d}$ & 146.3 & 132.8 & 138.1 & 123.1 & 150.5 & 163.5 & 138.4 & 121.6 & 137.5 & 87.7 \\
$\mathbf{4 e}$ & 146.4 & 132.9 & 138.1 & 123.1 & 150.5 & 163.5 & 137.5 & 121.1 & 128.7 & 127.7 \\
$\mathbf{4 f}$ & 146.4 & 133.0 & 138.1 & 123.1 & 150.5 & 163.4 & 135.0 & 115.4 & 121.3 & 158.4 \\
& & & & & & & & $J=22 \mathrm{~Hz}$ & $J=8 \mathrm{~Hz}$ & $J=241 \mathrm{~Hz}^{*}$ \\
$\mathbf{4 g}$ & 146.2 & 132.5 & 138.2 & 123.2 & 150.8 & 164.1 & 142.7 & 119.50 & 133.4 & 105.8 \\
\hline
\end{tabular}

${ }^{*} \mathrm{C}$-F couplings

\subsection{1-(2-Chloronicotinoyl)-2-(nitrophenyl)hydrazines}

The 1-(2-chloronicotinoyl)-2-(nitrophenyl)hydrazines 6a-c (Scheme 3) were synthesized and evaluated for their in vitro antibacterial activity against Mycobacterium tuberculosis $\mathrm{H}_{37} \mathrm{Rv}$ using the Alamar Blue susceptibility test and the activity expressed as the minimum inhibitory concentration (MIC) in $\mu \mathrm{g} / \mathrm{mL}$. However, none of the compounds above mentioned were active.<smiles>O=C(Cl)c1cccnc1Cl</smiles>

2-chloro-3pyridinecarbonyl chloride

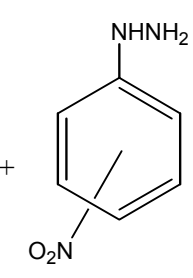

5a: $o$-nitro 5b: $m$-nitro 5c: $p$-nitro

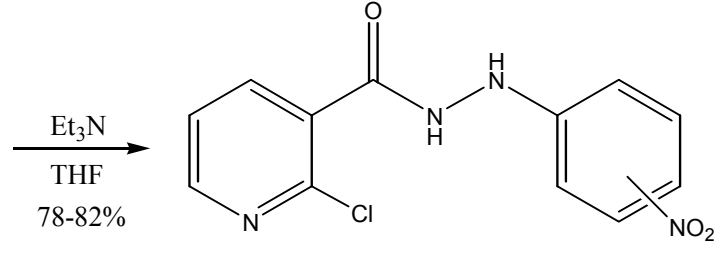

6a: $o$-nitro $\quad(78 \%)$

6b: $m$-nitro $(80 \%)$ 6c: $p$-nitro $(82 \%)$

\section{Scheme 3}

\section{2-Chloro- $N$-(2-pyridinyl)nicotinamides}

The 2-chloro- $N$-(2-pyridinyl)nicotinamides 8a-d (Scheme 4), were synthesized and its cyclization lead 5-oxo-5,6-dihydrodipyrido[1,2-a:3',2'-e]pyrimidin-11-ium chlorides 9a-d, which were identified by spectral data, which are presented in the Tables 4 and $\mathbf{5}$. The compounds 8a-d 
and 9a-d were evaluated for their in vitro antibacterial activity against Mycobacterium tuberculosis $\mathrm{H}_{37} \mathrm{Rv}$ using the Alamar Blue susceptibility test and the activity expressed as the minimum inhibitory concentration (MIC) in $\mu \mathrm{g} / \mathrm{mL}$. However, none of the compounds above mentioned were active.<smiles>O=C(Cl)c1cccnc1Cl</smiles>

2-chloro-3pyridinecarbonyl chloride<smiles>Cc1cccnc1N</smiles>

7a: 3-methyl

7b: 4-methyl

7c: 5-methyl

7d: 6-methyl

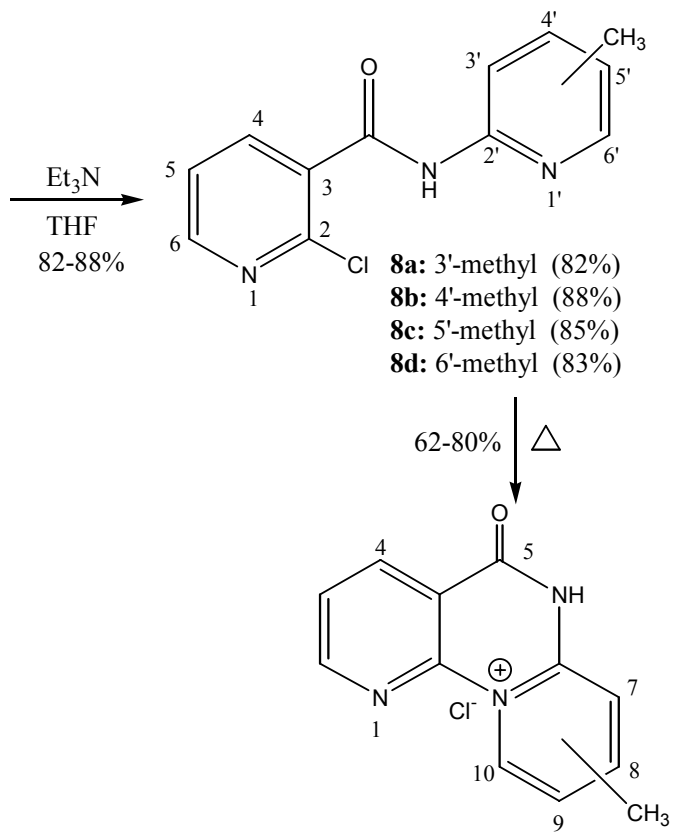

9a: 7 -methyl $(66 \%)$

9b: 8 -methyl $(80 \%)$

9c: 9 -methyl (77\%)

9d: 10-methyl (62\%)

\section{Scheme 4}

Table 4. ${ }^{1} \mathrm{H}-\mathrm{NMR}$ chemical shifts of compounds $\mathbf{8 a - d}$

\begin{tabular}{ccccccccc}
\hline & \multicolumn{7}{c}{$\delta{ }^{1} \mathrm{H}(\mathrm{ppm})$} \\
\hline Products & $\mathrm{NH}$ & $\mathrm{H}_{4}$ & $\mathrm{H}_{5}$ & $\mathrm{H}_{6}$ & $\mathrm{H}_{3}$, & $\mathrm{H}_{4}$, & $\mathrm{H}_{5}$, & $\mathrm{H}_{6}$, \\
\hline $\mathbf{8 a}$ & -- & 8.16 & 7.48 & 8.47 & -- & 7.82 & 7.32 & 8.24 \\
$\mathbf{8 b}$ & 11.25 & 8.19 & 7.48 & 8.45 & 7.60 & -- & 7.20 & 8.23 \\
$\mathbf{8 c}$ & 11.06 & 8.04 & 7.52 & 8.50 & 8.08 & 7.68 & -- & 8.20 \\
$\mathbf{8 d}$ & 11.21 & 8.03 & 7.52 & 8.50 & 8.00 & 7.74 & 7.05 & -- \\
\hline
\end{tabular}


Table 5. ${ }^{1} \mathrm{H}-\mathrm{NMR}$ chemical shifts of compounds 9a-d

\begin{tabular}{cccccccc}
\hline \multicolumn{7}{c}{$\delta{ }^{1} \mathrm{H}(\mathrm{ppm})$} \\
\hline Products & $\mathrm{H}_{2}$ & $\mathrm{H}_{3}$ & $\mathrm{H}_{4}$ & $\mathrm{H}_{7}$ & $\mathrm{H}_{8}$ & $\mathrm{H}_{9}$ & $\mathrm{H}_{10}$ \\
\hline 9a & 8.98 & 7.84 & 8.63 & -- & 7.79 & 7.05 & 9.32 \\
9b & 9.15 & 8.03 & 8.80 & 7.71 & -- & 7.64 & 9.80 \\
9c & 9.18 & 8.06 & 8.82 & 8.07 & 8.46 & -- & 9.78 \\
9d & 9.03 & 7.95 & 8.72 & 7.68 & 8.20 & 7.41 & -- \\
\hline
\end{tabular}

\section{Nitroisoniazid derivatives}

The $\quad N^{\prime}-[(E)$-(nitrophenyl)methylidene $]$ isonicotinohydrazides $\quad$ 11b-d $\quad$ (Scheme 5), were synthesized and identified by spectral data, which are presented in the Table 6. These compounds were evaluated for their in vitro antibacterial activity against Mycobacterium tuberculosis $\mathrm{H}_{37} \mathrm{Rv}$ using the Alamar Blue susceptibility test and the activity expressed as the minimum inhibitory concentration (MIC) in $\mu \mathrm{g} / \mathrm{mL}$ (Table 7). Depending on the position of the nitro group in the aromatic ring, different MIC values were obtained when compared with phenyl group, demonstrating the importance of the nitro group for the biological activity in this class of compounds. In ortho or meta position, MIC values were higher $(5.0 \mu \mathrm{g} / \mathrm{mL})$ when compared with the phenyl system $(3.2 \mu \mathrm{g} / \mathrm{mL})$. However, when the nitro group was placed in the para position it increases by three folds the biological activity, with MIC values of $1.2 \mu \mathrm{g} / \mathrm{mL}$. It can be observed some modifications in the anti-tubercular activity when $N^{\prime}-[(E)$ (nitrophenyl)methylidene]isonicotinohydrazides $\mathbf{1 1 b}-\mathbf{d}^{12}$ were compared with the $N^{r}-[(E)-$ (disubstituted-phenyl)methylidene]isonicotino-hydrazides 12a-c ${ }^{13}$ and $N^{\text {'- }}$ (disubstitutedbenzoyl)isoniazid 13a-c ${ }^{14}$ derivatives containing nitro groups, which were previously evaluate by our group (Scheme 6). For example, the $N^{\prime}$ (disubstitutedbenzoyl)isoniazid 13a-c were more activity when compared with isonicotinohydrazides 11b-d and 12a-c. The dinitro compound 12a and the nitrochloro compound 12b did not show increased anti-tubercular activity when compared with the mononitroisonicotinohydrazide 11d. However, the compound 12c, which has respectively, the nitro group and chloro atom at 2 and 3 positions in the aromatic ring presented increased anti-tubercular activity, when compared with the mononitroisonicotino-hydrazide 11b indicating that chloro atom at 3 position is important for the biological activity. 
<smiles>NNC(=O)c1ccncc1</smiles>

Isoniazid<smiles>Cc1ccc(C=O)cc1[N+](=O)[O-]</smiles>

10a: $o$-nitro 10b: $m$-nitro 10c: $p$-nitro

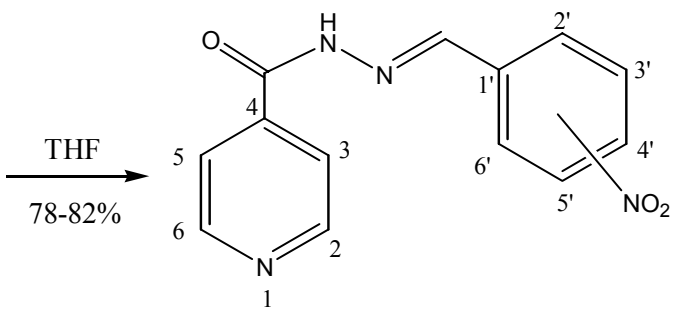

11a: phenyl $(80 \%)$

11b: $o$-nitro $(78 \%)$

11c: $m$-nitro $(80 \%)$

11d: $p$-nitro $\quad(82 \%)$

\section{Scheme 5}

Table 6. ${ }^{1}$ H-NMR chemical shifts of compounds 11a-d

\begin{tabular}{cccccccccc}
\hline & \multicolumn{8}{c}{$\delta{ }^{1} \mathrm{H}(\mathrm{ppm})$} \\
\hline Products & $\mathrm{NH}$ & $\mathrm{N}=\mathrm{CH}$ & $\mathrm{H}_{2,6}$ & $\mathrm{H}_{3,5}$ & $\mathrm{H}_{2}$, & $\mathrm{H}_{3}$, & $\mathrm{H}_{4}$, & $\mathrm{H}_{5}$, & $\mathrm{H}_{6}$, \\
\hline 11a & 12.10 & 8.47 & 8.79 & 7.83 & 7.76 & $7.47-7.57$ & $7.47-7.57$ & $7.47-7.57$ & 7.76 \\
\multirow{2}{11b}{} & 12.42 & 8.89 & 8.80 & $7.85-7.82$ & -- & $8.13-8.10$ & 7.71 & 7.71 & \\
& & & & & & & $7.85-7.82$ & $7.85-7.82$ & $8.13-8.10$ \\
11c & 12.34 & 8.58 & 8.81 & 7.84 & 8.58 & -- & 8.29 & 7.78 & 8.29 \\
& & & & & & & 8.19 & & 8.19 \\
11d & 12.37 & 8.57 & 8.81 & 7.84 & 8.02 & 8.32 & -- & 8.32 & 8.02 \\
\hline
\end{tabular}
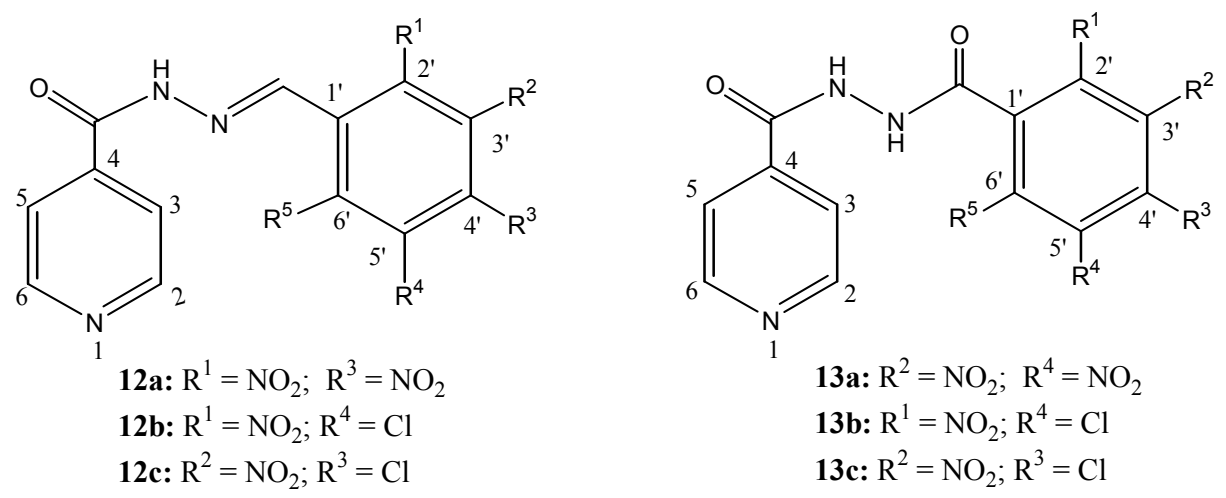

\section{Scheme 6}


Table 7. Antimycobacterial activities and $\log \mathrm{P}$ measurements of derivatives 11a-d, 12a-c and 13a-c

\begin{tabular}{ccc}
\hline Products & $\mathrm{MIC}(\mu \mathrm{g} / \mathrm{mL})$ & $\log \mathrm{P}^{\mathrm{a}}$ \\
\hline $\mathbf{1 1 a}$ & 3.12 & 1.810 \\
$\mathbf{1 1 b}$ & 5.00 & 1.720 \\
$\mathbf{1 1 c}$ & 5.00 & 1.744 \\
$\mathbf{1 1 d}$ & 1.20 & 1.768 \\
$\mathbf{1 2 a}$ & 5.00 & 1.655 \\
$\mathbf{1 2 b}$ & 5.00 & 2.374 \\
$\mathbf{1 2 c}$ & 1.20 & 3.664 \\
$\mathbf{1 3 a}$ & 2.00 & 0.480 \\
$\mathbf{1 3 b}$ & 0.50 & 1.199 \\
$\mathbf{1 3 c}$ & 0.50 & 1.199 \\
RIP & 1.0 & 2.788 \\
INH & 0.2 & -0.969 \\
\hline
\end{tabular}

${ }^{\text {a }}$ Calculated by http://www.molinspiration.com/

\section{Experimental Section}

General Procedures. Melting points were determined on a Buchi apparatus and are uncorrected. Infrared spectra were recorded on a Thermo Nicolet Nexus 670 spectrometer as potassium bromide pellets and frequencies are expressed in $\mathrm{cm}^{-1}$. Mass spectra (CG/MS) were recorded on a Agilent Tecnologies 6890 / 5972A mass spectrometer. NMR spectra were recorded on a Bruker Avance 500 spectrometer operating at $500.00 \mathrm{MHz}\left({ }^{1} \mathrm{H}\right)$ and $125.0 \mathrm{MHz}\left({ }^{13} \mathrm{C}\right)$, in deuterated dimethylsulfoxide. Chemical shifts are reported in $\mathrm{ppm}(\delta)$ relative to tetramethylsilane. Proton and carbon spectra were typically obtained at room temperature. For TLC, plates coated with silica gel were run in chloroform/methanol mixture and spots were developed in Ultraviolet. For column chromatographic purification under gravity, column grade silica gel 60 (0.063-0.200 mm mesh size) was employed.

Biological test. The antimycobacterial activities of compounds 2a-c; 4a-g; 6a-c; 8a-d; 9a-d; and 11a-d were assessed against M. tuberculosis ATTC $27294^{15}$ using the micro plate Alamar Blue assay (MABA) ${ }^{16}$. This methodology is nontoxic, uses a thermally-stable reagent and shows good correlation with proportional and BACTEC radiometric methods. ${ }^{17,18}$ Briefly, $200 \mu \mathrm{L}$ of sterile deionized water was added to all outer-perimeter wells of sterile 96 well plates (falcon, 3072: Becton Dickinson, Lincoln Park NJ) to minimize evaporation of the medium in the test wells during incubation. The 96 plates received $100 \mu \mathrm{L}$ of the Middlebrook 7H9 broth (Difco laboratories, Detroit, MI, USA) and a serial dilution of the compounds 2a-c; 4a-g; 6a-c; 8a-d; 
9a-d; and 11a-d were made directly on the plate. The final drug concentrations tested were 0.01 to $20.0 \mu \mathrm{L} / \mathrm{mL}$. Plates were covered and sealed with parafilm and incubated at $37^{\circ} \mathrm{C}$ for five days. After this time, $25 \mu \mathrm{L}$ of a freshly prepared 1:1 mixture of Alamar Blue (Accumed International, Westlake Ohio) reagent and 10\% tween 80 was added to the plate and incubated for $24 \mathrm{~h}$. A blue color in the well was interpreted as no bacterial growth, and a pink color was scored as growth. The MIC (Minimal Inhibition Concentration) was defined as the lowest drug concentration, which prevented a color change from blue to pink.

\section{Conclusions}

In conclusion, none of the nicotinic derivatives tested were active against the M. tuberculosis. In the case of the isoniazid derivatives containing nitro groups evaluated, the best result was the compound 11d $(1.2 \mu \mathrm{g} / \mathrm{mL})$ when compared with first line drugs as isoniazid (INH) and rifampin (RIP). It suggests that this class of compounds may be selectively targeted to M. tuberculosis growth, also considering that they were not cytotoxic to host cells at the same concentration and could be a good starting point to find new lead compounds.

\section{References}

1. Elguero, J. In Comprehensive Heterocyclic Chemistry II; Katritzky, A. R.; Rees, C. W.; Scriven, E. F. V., Eds.; Pergamon Press: Oxford, UK, 1996; Vol.3, p 70.

2. Merck Index, 11th Edition, 6435.

3. http://www.mayoclinic.com/health/niacin/NS patient-niacin

4. http://www.aafp.org/afp/981015ap/mayfield.html

5. De Souza, M. V. N. Recent Patents on Anti-Infective Drug Discovery 2006, 1, 33.

6. De Clercq, E. Biochim. Biophys. Acta-Mol.Basis Disease 2002, 258, 1587.

7. De Souza, M. V. N. Vasconcelos, T. R. A.; Wardell, S. M. S. V.; Wardell. J. L.; Low, J. N.; Glidewell, C. Acta Cryst. 2005, C61, 204.

8. Cuffini, S.; Glidewell, C.; Low, J. N.; De Oliveira A. G.; De Souza, M. V. N.; Vasconcelos, T. R. A. Wardell, S. M. S. V. Wardell, J. L. Acta Cryst. 2006, B62, 651.

9. Wardell, S. M. S. V.; De Souza, M. V. N.; Wardell, J. L.; Low, J. N.; Glidewell, C. Acta Cryst. 2007; B63; 101.

10. Fernandes, E. L.; Magalhães, A.; Paes, K. C.; De Souza, M. V. N.; Vasconcelos, T. R. A.; Wardell, J. L.; Wardell, S. M. S. V. J. Chem. Res. 2006, 93.

11. Wardell, S. M. S. V.; De Souza, M. V. N.; Wardell, J. L.; Low, J. N.; Glidewell, C. Acta Cryst. 2005, C61, 683.

12. Wardell, S. M. S. V.; De Souza, M. V. N.; Wardell, J. L.; Low, J. N.; Glidewell, C. Acta Cryst. 2007, $B$, In press (Forthcoming articles). 
13. Souza, M. V. N.; Vasconcelos, T. A.; Mello, S. C. P.; Wardell, S. M. S. V.; Peralta, M. A.; Ferreira, B.; Henrique, M. G. M. O.; Neves Junior, I.; Lourenço, M. C. S. Letters in Drug Design \& Discovery, 2005, 2, 451.

14. Junior, N. I.; Lourenço, M. C. S.; De Miranda, G. B. P.; Vasconcelos, T. R. A.; Pais, K. C.; Júnior, J. P. A.; Wardell, S. M. S. V.; Wardell, J. L.; De Souza, M. V. N. Letters in Drug Design \& Discovery, 2006, 3, 424.

15. Canetti, J., Rist, E.; Grosset, R. Pneumology 1963, 27, 217.

16. Franzblau, S. G.; Witzig, R. S.; McLaughlin, J. C.; Torres, P.; Madico, G.; Hernandez, A.; Degnan, M. T.; Cook, M. B.; Quenzer, V. K.; Ferguson, R. M.; Gilman, R. H. J. Clin. Microbiol. 1998, 36, 362.

17. Vanitha, J. D.; Paramasivan, C. N. Mycobacteriology 2004, 49, 179.

18. Reis, R. S.; Neves Jr., I.; Lourenço, S. L. S.; Fonseca, L. S.; Lourenço, M. C. S. J. Clin. Microbiol. 2004, 42, 2247. 